\title{
The development of a Line-to-Ground Fault Detection System for a DC feeding system on a support pole
}

\author{
M. Shimbo \\ East Japan Railway Company, Japan
}

\begin{abstract}
This paper presents the overview of a Line-to-Ground Fault Detection System of a DC feeding system on a support pole and some test results of the detection function. Sometimes, the voltage of lightning strikes can create closed circuits around insulators, and a DC feeding system is grounded if the supports of the overhead lines are made of steel. Existing protection systems on substations cannot detect the accident because they cannot distinguish between the current of train loads and the current of ground faults. Therefore the current of the ground fault makes an arc around the insulators of the overhead lines. The arc heats the overhead lines and they may be broken by tension. The breakage may occur within 3.1 seconds and there is no conclusive method to prevent the accident. Therefore, we developed a new system to prevent this kind of accident. A detection device having a wireless communication device for transmitting detected information is installed in each support pole. When the detection device senses the ground fault, the information is transmitted by multihop communication through some devices on support poles, and devices on substation open circuit breakers to terminate the ground fault. To detect the ground faults, Giant Magnetoresistance (GMR) sensors were selected. The test results confirmed that the sensors had enough performance against strong magnetic fields produced by the impulse current of lightning strikes. And the test results also confirmed that the sensors could detect 300A of the line-to-ground fault current on the support pole.
\end{abstract}

Keywords: line-to-ground fault, DC feeding system, magnetic field sensors, GMR. 


\section{Introduction}

On the DC feeding systems of East Japan Railway Company, sometimes the lightning strikes on supports of overhead catenaries broke the overhead lines, as shown in fig. 1. Fig. 2 shows an example of the accident which happened in Tokyo metropolitan area in 2008. Existing protection systems on substations cannot detect the accident because they cannot distinguish between current of train loads and current of ground faults. Matsumoto et al.[1] found that the breakage may occur within 3.1 seconds if the ground fault current is over 2000A. There is no conclusive method to detect the line-to-ground faults caused by lightning strikes on supports in such short time now. Thus the line-to-ground fault detection system has been needed.

Although arcing horns may be able to prevent arcs around insulators of overhead lines, a lot of arcing horns are needed because each single insulator supporting feeders and feeder messengers needs the arcing horns. And there are maintenance and cost issues. Therefore we developed the system as a new method which could prevent the accident at low total cost of ownership.

This paper presents the overview of the system, the function of line-to-ground fault detection and the results of some tests of the detection function.

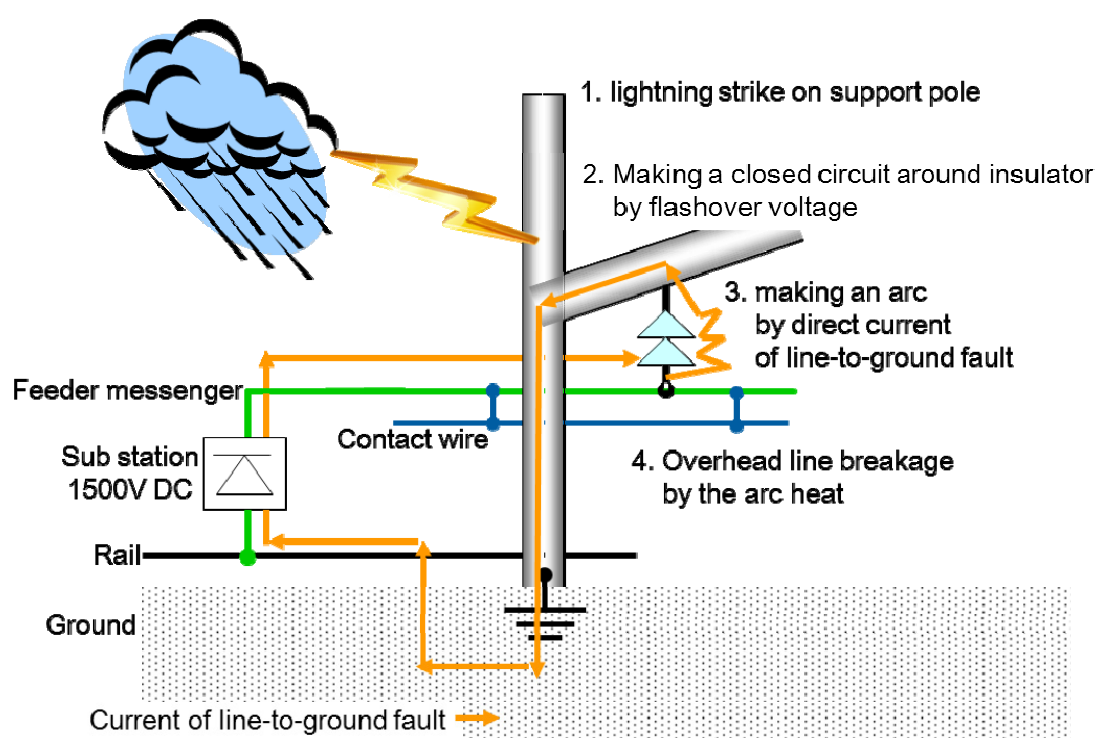

Figure 1: Overhead line breakage by lightning strike on support of overhead catenary on DC feeding system. 


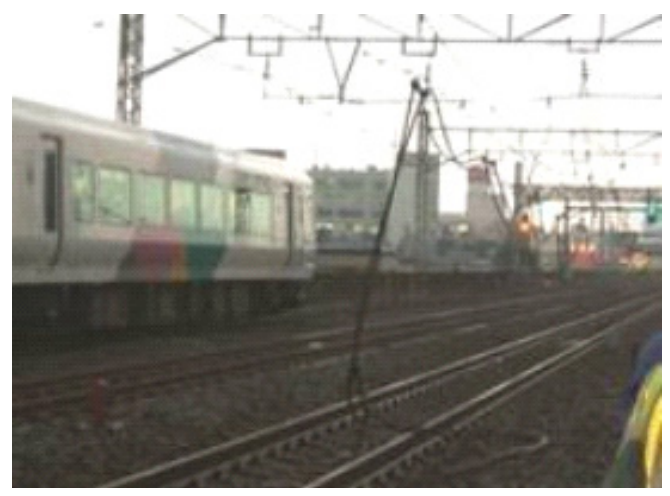

Figure 2: Overhead lines breakage by lightning strike in Tokyo metropolitan area in 2008.

\section{Overview of the Line-to-Ground Fault Detection System}

The system was designed for the overhead lines protection in viaduct section, as shown in fig. 3, to have three features which are an accident location detection, easy maintenance and easy construction. Detection devices having wireless communication devices for transmitting the detected information are put on the support poles. As shown in fig. 4, when a detection device senses ground fault, the information is transmitted by multihop wireless communication through some devices on support poles, and devices on substation open circuit breakers to terminate the ground fault current.

The system can identify on which support pole the ground fault occurred. And it is easy to put the devices on support poles. And maintenance is also easy by self-check function of the system. If the device was broken, the system could identify which device was broken.

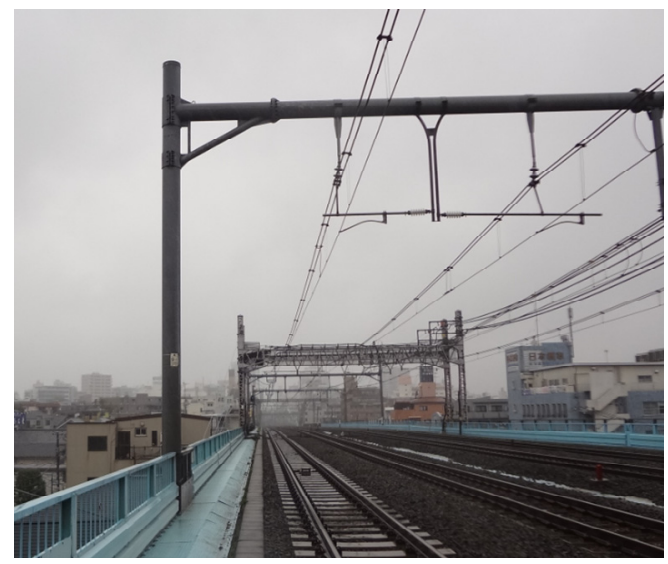

Figure 3: Example of overhead lines and supports in viaduct section. 


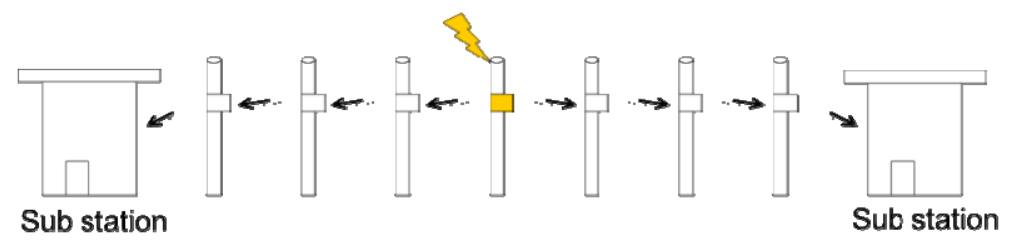

Figure 4: Example of multihop wireless communication to terminate the ground fault current by circuit breakers on the substations.

The device has three major functions which are detection, transmission and power supply. To detect ground fault current on the support pole, GMR sensors were selected. To transmit information, multihop wireless communication was selected. As power supply, solar cells and lead-acid storage batteries were selected. Fig. 5 shows the appearance of the devices.

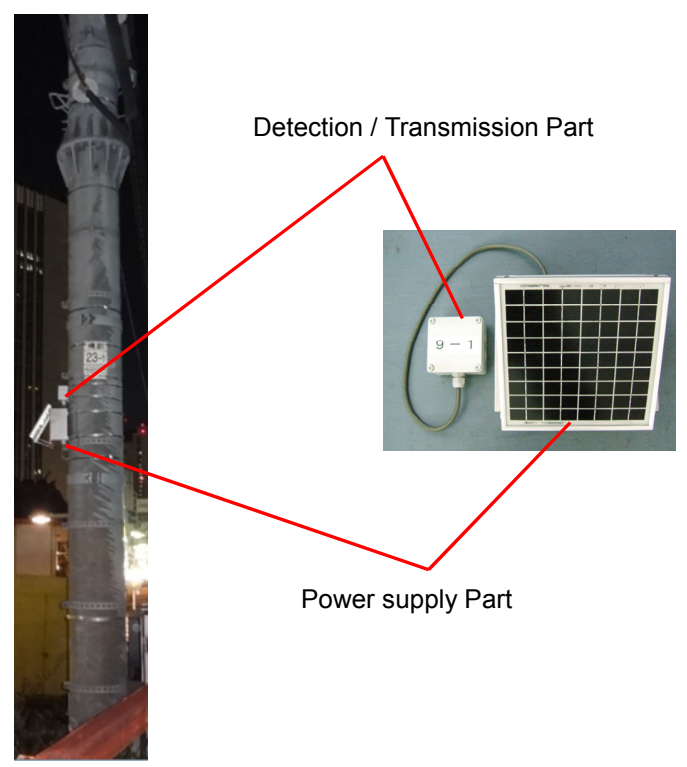

Figure 5: Appearance of developing devices.

\section{Detection function}

\subsection{Detection theory}

There are two types of systems to detect current generally. Those are voltage detection systems and magnetic field detection systems. The developed system must have impulse current resistance by lightning strikes on supports. In case of selecting voltage detection systems, high voltage resistant design is needed and it 
will be expensive. And magnetic field detection systems which can measure magnetic field by a non-contact don't need high voltage resistant design, so magnetic field detection systems were more suitable to the developed system if they could tolerate impulse current on the pole and detect ground fault current.

Fig. 6 shows the ground fault detection by magnetic field sensor. The ground fault current produces the magnetic field as Ampère's circuital law. And ground fault current is calculated by eqn. (1) as Ampère's circuital law too.

$$
B=\mu_{0} \frac{I}{2 \pi r}(\mathrm{~T})
$$

Putting the magnetic field sensor on the pole enables to detect the ground fault.

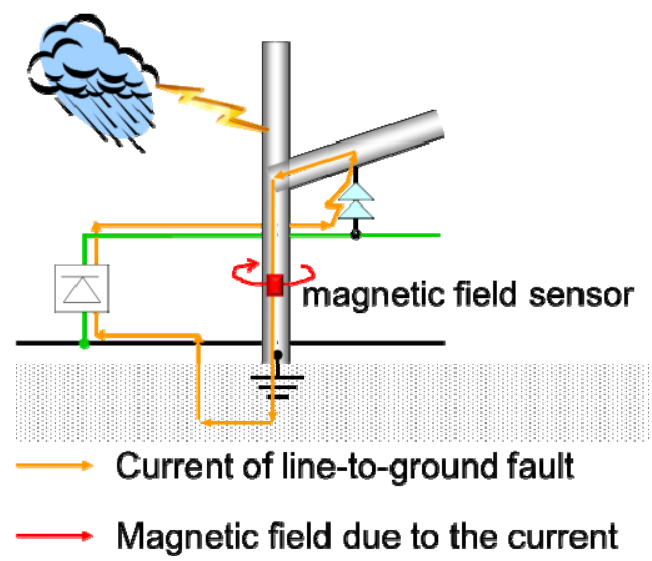

Figure 6: Ground fault detection by magnetic field sensor.

\subsection{Test results}

\subsubsection{Test plan summary}

Among some candidates, GMR sensor (AAL002-02, NVE [2]) was selected by desk research because it has high sensitivity and reasonable price. In order to evaluate the appropriate, sensors were tested by impulse withstand performance tests and trial tests simulating ground fault currents on the support pole.

\subsubsection{Impulse withstand test}

An impulse withstand test simulated strong magnetic fields produced by impulse current of lightning strikes. If distance between the center of a support pole and the sensor is $197.8 \mathrm{~mm}$, putting neodymium magnet which have $250-300 \mathrm{mT}$ near the sensor enables to simulate 247-297kA of impulse current on the support pole. The distance simulates putting the detecting part on the surface of the mast 
( $\$ 355.6 \mathrm{~mm})$. And the current is calculated by eqn. (1) as Ampère's circuital law. Table 1 shows comparing the output of the sensor before putting the magnet and after by known magnetic fields. The sensor output differences were negligible, so it was confirmed that the sensors had enough performance against the strong magnetic fields produced by impulse current of the lightning strikes.

Table 1: Comparing the outputs of GMR sensor before/after putting the magnet.

\begin{tabular}{|c|c|c|c|c|}
\hline \multirow{2}{*}{$\begin{array}{c}\text { magnetic } \\
\text { field }\end{array}$} & \multicolumn{3}{|c|}{ sensor output $(\mathrm{mV})$} & different ratio \\
\cline { 2 - 4 } & before & after & difference & $0.52 \%$ \\
\hline A & 965 & 960 & 5 & $0.49 \%$ \\
\hline B & 1025 & 1020 & 5 & $0.49 \%$ \\
\hline C & 1025 & 1020 & 5 & $-0.51 \%$ \\
\hline E & 980 & 985 & -5 & $0.20 \%$ \\
\hline F & 1022 & 1020 & 2 & $0.20 \%$ \\
\hline G & 1022 & 1020 & 2 & \\
\hline
\end{tabular}

\subsubsection{Trial test simulating ground fault current on the support pole}

All magnets and magnetic materials have magnetic hysteresis. Hysteresis refers to the history of the magnetic field applied to the material. The GMR sensors (AAL002-02, NVE) are made of magnetic materials, so they are subject to hysteresis effects. And directions of impulse current by lightning strikes are possibly both directions, the developed system needed bipolar operation. Hysteresis is much more exaggerated in the sensor element when a bipolar magnetic field is applied to the sensor. So the following test was done to evaluate hysteresis effects to the sensor.

Fig. 7 shows the measurement configuration. The sensor location was $20 \mathrm{~mm}$ from the surface of the mast $(\$ 355.6 \mathrm{~mm})$ which is used as a support pole, the distance between the sensor location and the center of the mast was $197.8 \mathrm{~mm}$. In this configuration, $3000 \mathrm{~A}$ of $\mathrm{DC}$ equals to $3 \mathrm{mT}$ of magnetic field around the sensor.

Fig. 8 shows the output characteristic of the sensor. The horizontal axis was converted from DC to magnetic flux density by eqn. (1). Hysteresis is confirmed on fig. 8 .

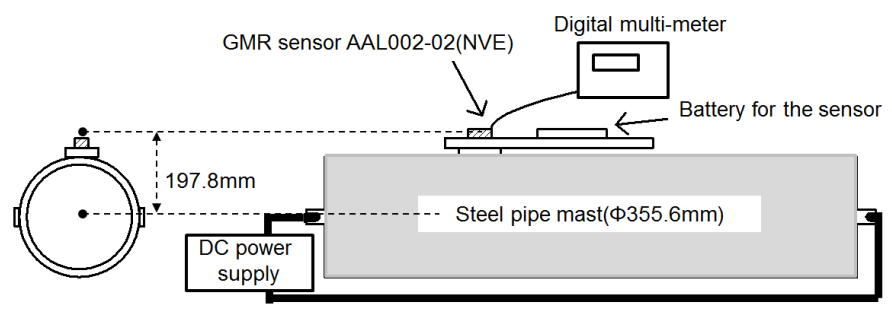

Figure 7: Measurement configuration of trial test simulating ground fault current on the support pole. 


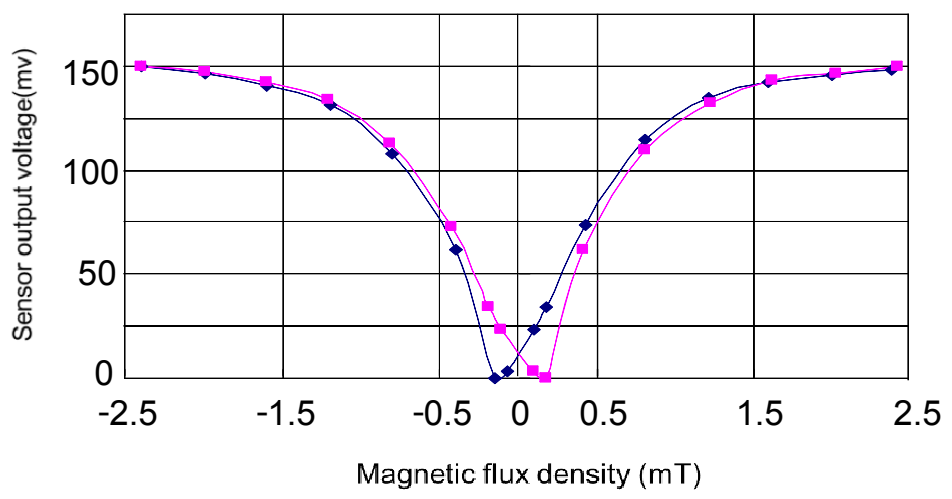

Figure 8: Output characteristic of GMR sensor AAL002-02(NVE).

Fig. 9 is an enlargement of fig. 8's part and shows the offset and threshold setting of the sensor. Setting offset and buffer enables to avoid effect of hysteresis and detecting errors. According to NVE's data sheet [2], output voltage changes of the sensors due to temperature changes are negligible in the area to consider. Shimbo et al. [3] found that the steel pipe masts had $0.05 \mathrm{mT}$ of magnetic flux density by residual magnetization on their level surfaces. And the magnetic flux density by train loads was negligible as long as putting the sensor on the middle of support pole in a direction parallel to the overhead lines. To set the threshold of the detection, some buffer is needed for residual magnetization of steel pipe masts and individual differences of the sensors. The offset value is $0.21 \mathrm{mT}$ from fig. 8 and the threshold can be set $0.3 \mathrm{mT}$ adding $0.09 \mathrm{mT}$ as the buffer. The threshold is able to detect 300A of grand fault current on steel pipe mast $(\$ 355.6 \mathrm{~mm})$. The developing system was designed for the viaduct section, grounding resistance of support poles is less than $5 \Omega$ there and other resistances are small enough to neglect. The feeding voltage is $1500 \mathrm{~V}$, therefore $300 \mathrm{~A}$ is sufficient to detect the grand fault. Consequently the threshold was reasonable and also the sensors were suitable to the developed system.

In order to eliminate the unnecessary operations of the circuit breakers on substations by the developed system, the system should be able to distinguish between ground fault current and impulse current due to lightning strikes. The detection part of the system was designed to distinguish them by judging their continuation time.

\section{Conclusion}

In this paper the overview of a Line-to-Ground Fault Detection System of a DC feeding system on support poles and some test results of a detection function are presented. Detection devices having wireless communication devices for transmitting the detected information are put on the support poles supporting overhead lines. When one detection device senses ground fault, the information is transmitted by multihop communication through some devices on support 


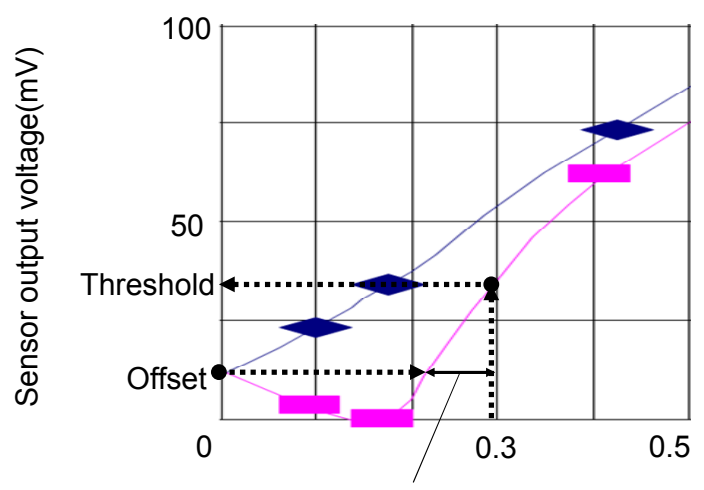

buffer for residual magnetization of steel pipe masts and individual differences of the sensors

Magnetic flux density (mT)

Figure 9: Offset and threshold setting of GMR sensor AAL002-02(NVE) from the test result.

poles, and devices on substation open circuit breakers. To detect line-to-ground faults, GMR sensors (AAL002-02, NVE) were selected. The test results confirmed that the sensors had enough performance against the strong magnetic fields produced by impulse current of lightning strikes. And the test results also confirmed that the sensors could detect $300 \mathrm{~A}$ of the line-to-ground fault current. Therefore the sensors were suitable to the system.

\section{References}

[1] Matsumoto, A., Uematsu, S., Yamamoto, H., Negishi, H., Mitoma, Y., Verification test of disconnecting phenomenon on feeder messenger caused by continuous current. National Convention Record IEE Japan, 5th fascicle, pp. 167-168, 2013.

[2] NVE corporation, http://www.nve.com/Downloads/analog_catalog.pdf

[3] Shimbo, M., Kuraoka, T., Iwainaka, A., Nagamori, M., Magnetic density measurement around pillars of DC feeding system. National Convention Record IEE Japan, 5th fascicle, pp. 243-244, 2013. 